\title{
Characterization of Cardiac Troponin I Raman Signature in Bovine Serum Albumin and Human Blood Serum for the Potential Diagnosis of Myocardial Infarction
}

Sara Moghaddamjoo ${ }^{*}$, Amir Pasha Tashakor ${ }^{1}$, Sahba Talebi Fard ${ }^{1}$, Yashar Tashakkor A ${ }^{2,3}$, Ezra Kwok ${ }^{1}$, Yueh Alison Li ${ }^{5}$, Najme Tavassoli ${ }^{4}$, Glen F Tibbits ${ }^{5}$, Grant Ed ${ }^{4}$ and Andrew Rawicz ${ }^{3}$

${ }^{1}$ Biomedical Engineering Program, Faculty of Applied Science, University of British Columbia, Vancouver, British Columbia, Canada ${ }^{2}$ Department of Medicine, University of British Columbia, Vancouver, British Columbia, Canada

${ }^{3}$ Division of Biomedical Engineering, School of Engineering Science, Simon Fraser University, Vancouver, British Columbia, Canada

${ }^{4}$ Department of Chemistry, Faculty of Science, University of British Columbia, Vancouver, British Columbia, Canada

${ }^{5}$ Department of Biomedical Physiology and Kinesiology, Simon Fraser University, Vancouver, British Columbia, Canada

\begin{abstract}
Cardiac troponin I is one of the most specific and widely used biomarkers of myocardial infarction. Prompt recognition of elevated troponin concentrations in the blood is essential to the diagnosis and management of acute coronary syndrome (ACS). Raman spectroscopy allows for detection of the unique cTnl Raman signature based on quantitative measurement of wavelength and intensity of in elastically scattered light from molecules. For the first time in the literature, we have successfully characterized and verified the unique Raman signature of mouse cardiac troponin I protein in Bovine Serum Albumin and Human Blood Serum. Our findings potentiate technological advancement towards point-of-care testing measurement of cardiac biomarkers, which can be employed, potentially more rapid than conventional ELISA assays, in the outpatient setting and emergency departments for routine detection of cardiac troponin I elevation from patients suspected of suffering from acute coronary artery syndrome.
\end{abstract}

Keywords: Cardiac biomarker; Raman spectroscopy; Myocardial infarction; Biophotonics; Point of care testing; Acute coronary syndrome

\section{Introduction}

Detection of cardiac biomarkers in addition to characteristic symptoms and electrocardiographic abnormalities constitute current diagnostic guidelines for Acute Coronary Syndrome (ACS) [1]. An elevation in concentrations of the protein biomarker, cardiac troponin I (cTnI), has diagnostic, management, and prognostic implications $[2,3]$ and is routinely quantified in emergency departments. In symptomatically identical patients, an elevation in cTnI concentration may warrant patient hospitalization, immediate catheter angiography, and possible therapeutic intervention, as opposed to supportive management and potential same day discharge [4]

Despite their recognition as the current gold standard [5], limitations exist in detecting elevated myocardial infarction biomarker concentrations [6] such as the need to obtain blood samples, lack of inter-laboratory standardization, and time delays that can surpass an hour for laboratory processing. Assay sensitivity in current laboratory-based automated platforms limits detection of circulatory cTnI concentrations to post symptomatic levels [7], despite systemic circulation at lower concentrations several hours prior. Moreover, population based observational studies in patients suspected of ACS exhibit prevalent hesitation to promptly contact primary care physicians, as well as delayed hospital presentation as late as a median of 4 hours after symptom onset [8,9]. Unfortunately, comparatively higher myocardial infarction biomarker concentrations suggest prolonged ischemia and irreversible cardiac damage.

Prompt diagnosis and intervention is the cornerstone of management in ACS, and advances in diagnostic technology using animal and human models are recently emerging [7,10,11]. Raman spectroscopy is based on quantitative measurement of wavelength and intensity of in elastically scattered light from molecules. The Raman scattered light occurs at wavelengths that are shifted from the incident light by energies of molecular vibrations. This vibration can be observed in either the Infrared (IR) or Raman spectra. While IR spectroscopy measures the absorption of infrared light by the sample, Raman spectroscopy measures the scattered light.

The objective of this study is to utilize biophotonic technology to characterize the unique Raman signature of recombinant mouse cTnI in Bovine Serum Albumin (BSA) and Human Blood Serum (HBS). The importance of selecting recombinant mouse cTnI is the striking structural similarities between human and mouse cTnI [12]. The potential clinical application of this physiological signature, as governed by its distinct molecular structure, is to serve as a highly specific point-of-care testing (POCT) for prompt diagnosis of ACS.

\section{Research Significance}

Despite medical breakthroughs in the management of cardiovascular diseases, Coronary Artery Disease (CAD) remains a global leading cause of mortality [13]. Alarming statistics persist despite widespread implementation of interdisciplinary public health measures aiming at the prevention and early treatment of heart diseases. Particular vulnerability of myocardial cells to inadequate blood perfusion and the critical role of the heart as a central organ translate into ischemic-reperfusion injury and significant morbidity and mortality from myocardial infarction (MI). The spectrum of pathophysiological changes associated with ACS commence hours prior to objective symptoms as specific myocardial infarction

*Corresponding author: Sara Moghaddamjoo, Biomedical Engineering Program Faculty of Applied Science, University of British Columbia, Vancouver, British Columbia, Canada, E-mail: sara.moghaddamjoo@gmail.com

Received December 12, 2015; Accepted December 19, 2015; Published Decembe 22, 2015

Citation: Moghaddamjoo S, Tashakor AP, Fard ST, Tashakkor AY, Kwok E, et al. (2015) Characterization of Cardiac Troponin I Raman Signature in Bovine Serum Albumin and Human Blood Serum for the Potential Diagnosis of Myocardial Infarction. Nat Prod Chem Res 4: 200. doi:10.4172/2329-6836.1000200

Copyright: ( 2015 Moghaddamjoo S, et al. This is an open-access article distributed under the terms of the Creative Commons Attribution License, which permits unrestricted use, distribution, and reproduction in any medium, provided the original author and source are credited. 
Citation: Moghaddamjoo S, Tashakor AP, Fard ST, Tashakkor AY, Kwok E, et al. (2015) Characterization of Cardiac Troponin I Raman Signature in Bovine Serum Albumin and Human Blood Serum for the Potential Diagnosis of Myocardial Infarction. Nat Prod Chem Res 4: 200. doi:10.4172/2329-6836.1000200

Page 2 of 5

biomarkers are released into the systemic circulation secondary to cardiac myocyte ischemia and lysis. Severe ischemia lasting as short as minutes followed by reperfusion may lead to myocyte necrosis and irreversible damage. Rapid detection of MI biomarkers with results available in minutes permits prompt decision-making and better patient management, particularly in cases with inconclusive findings from electrocardiography [8]. Considering that early medical intervention during ischemic attacks to the heart is of paramount importance and can prevent further tissue damage, early detection of such biomarkers can be lifesaving.

This paper briefly describes the principles behind Raman Spectroscopy and our experimental approach, followed by an illustration of the unique cTnI Raman signature, and a discussion of the findings.

\section{Methods and Materials}

\section{Raman spectroscopy}

In Raman spectroscopy, a laser beam is used to irradiate a sample over the Ultra-Violet-visible (UV-visible) and near-infrared-region (NIR) spectrum (200 to $900 \mathrm{~nm}$ ). The scattered beams are also at the UV-visible-NIR region and consist of two parts:

1. Rayleigh scattering which is strong and has the same frequency as the incident beam; and

2.

Raman scattering, which is very weak and has a frequency of higher or lower than the incident beam.

These discrepancies in frequency of incident beam and Raman scattering are unique to each molecule, enabling the identification of a molecule from its Raman signature. A Stokes shift is defined as a Raman scatter frequency that is lower than the incident beam frequency. Similarly, an Anti- Stokes is a Raman scatter frequency, higher than the initial incident beam.

Raman spectroscopies are done in the UV-visible and NIR region. Therefore, IR and Raman are considered complementary in that certain vibrations will be solely Raman active (e.g., totally symmetric vibrations), while others being exclusively IR active. Covalent bonds are generally very strong in Raman while ionic bonds are strong in IR. This feature of Raman makes it especially advantageous for our studies of proteins as it can adequately detect the vibration of the covalent bonds between amino acids. Another advantage of Raman over IR spectroscopy is that most proteins in the body are in an aqueous solution and water is inconveniently a strong absorber of IR. In contrast, water is a weak Raman scatterer. Raman scattering can be done in aqueous solutions using a single recording and it is not necessary to change gratings, beam splitters, filters and detectors in obtaining a Raman spectrum over a wavenumber range as wide as from $4000 \mathrm{~cm}^{-1}$ to $50 \mathrm{~cm}$ ${ }^{1}$. The reason being both the incident and the scattered beams are in the UV-Visible-NIR range of 200-900 $\mathrm{nm}$. Therefore, Raman spectroscopy in the $200-900 \mathrm{~nm}$ wavelength range can be manipulated by a single set of optical elements. Furthermore, water is relatively transparent to light in all or most of this wavelength range and this inherent quality makes Raman a superior choice for most bio scientific applications.

\section{Identifying the Raman signature of $\mathrm{cTnI}$ using a partial least square regression (PLSR) model}

Identifying analyte concentrations from an optical signal may require a simple linear model, such as models used in Evanescent Field sensors, where a clear linear relationship is observed [14,15]. However, in most cases of label-free detection in which a signature of a molecule at low concentration is being detected (absorption, reflection, or Raman spectroscopy) and other molecules with interfering signatures also exist, a linear relationship may not be as apparent. In such cases, regression analysis and sometimes noise reduction are required to identify the dependency and extract the correlation $[16,17]$. Inverse Least Square (ILS) methods, such as multiple linear regression (MLR), principal component regression (PCR), and partial least square (PLS), are commonly used and are suitable for spectroscopy signals. MLR finds factors that can correlate the Raman spectra with concentration. PCR finds factors with most variance in Raman spectra. However, we need to find wave numbers that capture the most variance in the Raman spectra and have the strongest correlation with cTnI concentrations. Because PLS is a combination of MLR and PCR, it is able to find the factors that can both capture variance in Raman spectra and achieve strong correlations between Raman spectra and cTnI concentrations. Therefore, the Partial Least Square Regression (PLSR) model is most effective method for our application.

Identification of the dependency between cTnI concentrations and its Raman spectra was achieved by using a PLSR model, which captures maximum variations in Raman spectra that best explains variations in cTnI concentration [11]. PLSR reduces the dimensionality of the system to a given number of components or latent variables. For each of these latent variables, a weight vector that describes the dependency of that component to the original variables in the Raman spectra is calculated. The PLSR weights manifest how strongly each variable in the Raman spectra can explain the variation in concentrations and therefore, the PLSR weight is a measure of variations in the signal that correlates strongly with a particular concentration $[18,19]$.

Each latent variable makes a contribution to the model. To evaluate the contribution of each latent variable, one quick measure is to calculate the percentage variance of the concentrations explained with the addition of each latent variable. Afterwards, the weight vector corresponding to the latent variable that explains the most variations has the indication of the strongest correlation between Raman spectra and the corresponding cTnI concentration. Moreover, preprocessing data with baseline correction and removing orthogonal noises reduces the complexity of the model, meaning that the weights corresponding to a smaller LV number explains stronger correlation with the concentrations and the corresponding wave numbers. In our application, for each set of data, we first identified the contribution of each regression component in explaining the variations in concentrations, and subsequently plotted the weights corresponding to the component with maximum contribution. That weight vector will manifest as the segments of the Raman spectrum that have the strongest correlation with $\mathrm{cTn}$ I concentrations.

\section{Recombinant mouse cTnI preparation}

Protein over-expression of recombinant mouse cTnI was performed as previously described [20]. In summary, recombinant mouse cardiac protein subunits were expressed in E. coli. BL21 (DE3) (Novagen) cells with the cTnI gene in the kanamycin resistant pSBET vectors. Our method of cTnI purification has also been previously reported $[20,21]$ with the following modifications: the cTnI- containing cell lysate was made to $25 \%$ saturation with ammonium sulphate, and the supernatant was obtained after centrifugation at 27,000 g for 30 minutes (Beckman JLA16.25) at $4^{\circ} \mathrm{C}$. The supernatant was dialyzed overnight against 2-3 changes of four liters of buffer A (6 M Urea, $25 \mathrm{mM}$ TEA-HCl, $1 \mathrm{mM}$ EDTA, and $1 \mathrm{mM} \mathrm{DTT}$ at $\mathrm{pH}$ 7.5) and then loaded onto a CM sepharose ${ }^{\circledast}$ column (XK26, Amersham Biosciences), which was 
Citation: Moghaddamjoo S, Tashakor AP, Fard ST, Tashakkor AY, Kwok E, et al. (2015) Characterization of Cardiac Troponin I Raman Signature in Bovine Serum Albumin and Human Blood Serum for the Potential Diagnosis of Myocardial Infarction. Nat Prod Chem Res 4: 200. doi:10.4172/2329-6836.1000200

previously equilibrated with buffer A. Cardiac TnI was eluted at 50$60 \%$ of a linearly increasing gradient of $0-0.5 \mathrm{M} \mathrm{NaCl}$ in buffer $\mathrm{B}(6 \mathrm{M}$ urea, $25 \mathrm{mM}$ TEA- $\mathrm{HCl}, 1 \mathrm{mM}$ EDTA, $1 \mathrm{mM}$ DTT and $0.5 \mathrm{M} \mathrm{NaCl}$ at $\mathrm{pH} 7.5$ ). If the purity was not satisfactory as determined by $12 \%$ Sodium dodecyl sulphate-polyacrylamide gel electrophoresis (SDS-PAGE), all fractions containing cTnI were pooled together and further purified by DEAE fast flow column (GE Healthcare). The fractions containing cTnI were dialyzed against buffer C (6 M urea, $20 \mathrm{mM}$ Tris- $\mathrm{HCl}, 1$ $\mathrm{mM}$ EDTA, and $0.5 \mathrm{mM}$ DTT at $\mathrm{pH}$ 8.0). Other contaminant proteins remained bound to the column while cTnI eluted from the flow through and wash fraction. CTnI was then dialyzed extensively against $5 \mathrm{mM}$ ammonium bicarbonate, lyophilized, and stored at $-20^{\circ} \mathrm{C}$.

The Raman spectra of two sets of analytes are measured and analyzed:

1. cTnI in BSA, $\mathrm{HCl}$ and $\mathrm{CTNC}$

2. cTnI in human blood serum

\section{Results}

For each set of data, we first identified the contribution of each regression component in explaining the variations in concentrations, and then plotted the weights corresponding to the component with maximum contribution. That weight vector indicates the segments of Raman spectra that have the strongest correlation with cTnI concentrations.

\section{cTnI signature}

Figure 1 illustrates the percentage variation of $\mathrm{cTnI}$ concentration explained versus the number of PLSR components or latent variables, both for the case of BSA and human blood serum. In the spectra for cTnI in BSA without preprocessing, the fourth LV explains the maximum variations, whereas the first latent variable would include the maximum explanation after data preprocessing to remove noise and baseline variations. After data preprocessing with baseline correction and OSC (Orthogonal Signal Correction - a method allowing for the elimination of background noise), the first latent variable explains more than $97.8 \%$ of the variance in concentrations.

\section{cTnI in BSA and in serum}

Figure 2 shows the PLSR weights for the Raman spectra of cTnI in BSA corresponding to their most significant latent variable concluded from Figure 1a. Figure 2a shows the weight for data without preprocessing and its most significant latent variable $\left(4^{\text {th }} \mathrm{LV}\right)$. Figure $2 \mathrm{~b}$ shows the weight vector for the $4^{\text {th }} \mathrm{LV}$, after the Raman signal is smoothed and corrected for baseline variations. Figure $2 \mathrm{c}$ shows the weight for data after preprocessing and its most significant latent variable $\left(1^{\text {st }} \mathrm{LV}\right)$.

As can be seen in the PLSR weights for $\mathrm{CTnI}$ in BSA without any preprocessing, peaks around wave numbers $1595 \mathrm{~cm}^{-1}$ and $1790 \mathrm{~cm}^{-1}$ are observed. However, they are masked by significant noise. After preprocessing data and removing orthogonal noises, two obvious peaks appear in the weighted signal (Figures 2 and 3). In the following section, it will be shown that these same peaks are observed in the other set of data with cTnI in blood serum.

Figure 3 shows the PLS weights for Raman spectra of the second set of data cTnI in Blood Serum corresponding to their most significant latent variable concluded from Figure $1 \mathrm{~b}$. Figure $3 \mathrm{a}$ shows the weight for data without preprocessing and its most significant latent variable $\left(2^{\text {nd }} \mathrm{LV}\right)$. This set of Raman spectra exhibited a lot of baseline

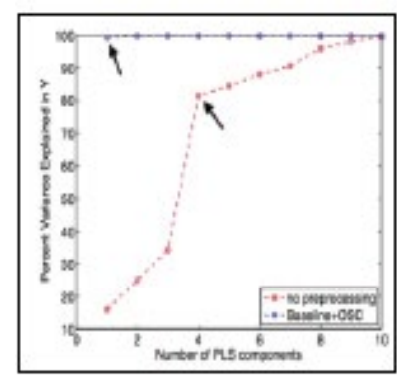

Figure 1.

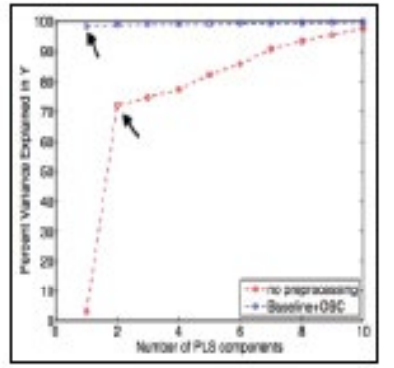

Figure 1b
Figure 1: Example Percent variation in concentration explained as a function of number of latent variables in the model. The arrows indicate the latent variable that explains most of the variations. (a) $\mathrm{cTnl}$ in $\mathrm{BSA}, \mathrm{HCl}$, and $\mathrm{cTnC}$; (b) cTnl in human blood serum.

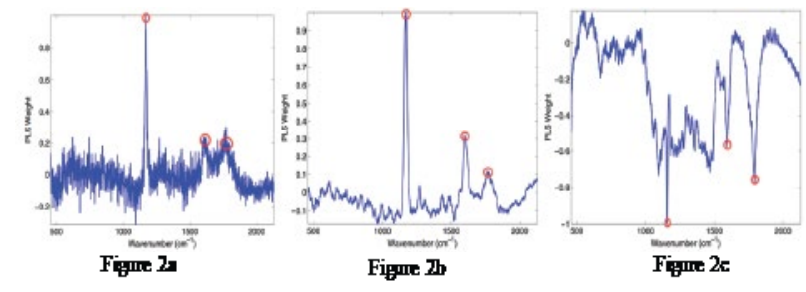

Figure 2: PLS weights of the most significant, LV for the Raman spectra of cTnl in BSA. The red circles from left to right correspond to the wavenumbers 1160 $\mathrm{cm}^{-1}, 1595 \mathrm{~cm}^{-1}, 1790 \mathrm{~cm}^{-1}$, respectively. (a) PLS weights of $4^{\text {th }} \mathrm{LV}$, for the raw data; (b) PLS weights of $4^{\text {th }}$ LV, for the smooth and baseline corrected data; (c) PLS weights of $1^{\text {st }} \mathrm{LV}$, for the preprocessed data.

variations. Therefore, correcting for baseline variations and smoothing the signal, the weight vector for the second latent variable can be found and is shown in Figure $3 \mathrm{~b}$. Figure $3 \mathrm{c}$ shows the weight for data after preprocessing and its most significant latent variable $\left(1^{\text {st }} \mathrm{LV}\right)$.

We have measured the Raman spectrum of cTnI in BSA. Reviewing the PLSR weight in all those measurements suggests a strong correlation between the existence of $\mathrm{cTnI}$ and certain wave numbers in the Raman spectrum. Thus, cTnI appears to have a Raman signature at the approximate wave numbers: $1160 \mathrm{~cm}^{-1}, 1595 \mathrm{~cm}^{-1}, 1790 \mathrm{~cm}^{-1}$, and potentially at the approximate wave numbers $1095 \mathrm{~cm}^{-1}, 1487 \mathrm{~cm}^{-1}$, $1524 \mathrm{~cm}^{-1}, 1630 \mathrm{~cm}^{-1}$. In order to verify the Raman signature, the PLS weights for both sets of cTnI in BSA and cTnI in serum data, with and without preprocessing, are plotted in Figure 4 to illustrate how they all show a strong correlation with similar wave numbers in Raman spectra. All these spectra reveal the existence of a Raman signature at the following wave numbers: $1160.8 \pm 5.4 \mathrm{~cm}^{-1} ; 1596.4 \pm 7.3 \mathrm{~cm}^{-1}$; $1790.2 \pm 11.1 \mathrm{~cm}^{-1}$.

\section{Discussion}

\section{Unique findings and clinical applications}

To the best of our knowledge, this is the first study that has successfully characterized and verified the unique Raman signature of mouse $\mathrm{cTnI}$ protein in BSA and human blood serum. $\mathrm{cTnI}$ is considered to be one of the most specific and widely used cardiac biomarkers [10] for myocardial infarction. The characteristic Raman signature allows for high specificity. Further optimization of our detection apparatus may improve the sensitivity of the cTnI measurement. Advancement in biomedical engineering, coupled with an incentive towards developing non-invasive and expeditious diagnostic devices, has allowed for the 


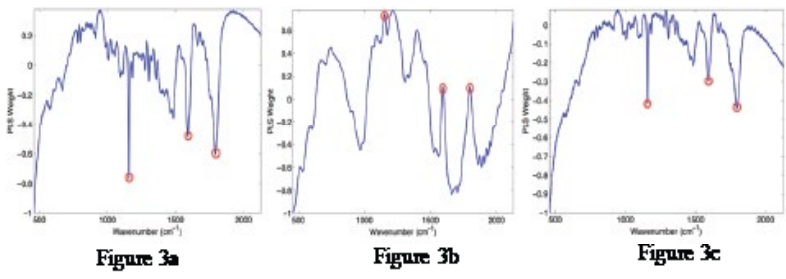

Figure 3: PLS weights of the most significant, LV for the Raman spectra of $\mathrm{cTnl}$ in human blood serum. The red circles from left to right correspond to the wavenumbers $1160 \mathrm{~cm}^{-1}, 1595 \mathrm{~cm}^{-1}, 1790 \mathrm{~cm}^{-1}$, respectively. (a) PLS weights of the $2^{\text {nd }} L V$, for the raw data; (b) PLS weights of the $2^{\text {nd }} L V$, for the smooth and baseline corrected data; (c) PLS weights of the $1^{\text {st }} \mathrm{LV}$, for the preprocessed data.

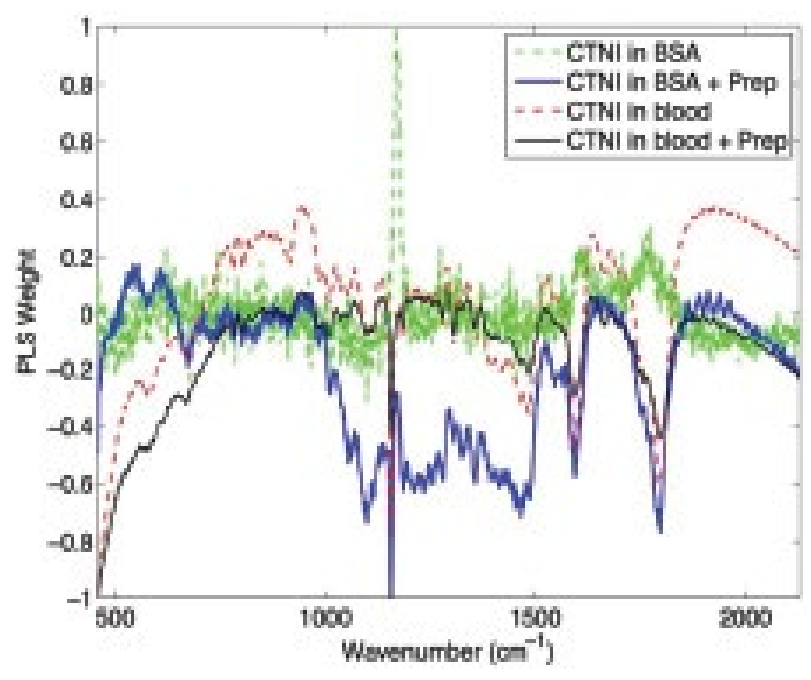

Figure 4: PLS weights for all sets of data, without and with preprocessing

inception of point-of-care testing (POCT) devices for diabetes mellitus [22], skin cholesterol measurement [23], and some novel cardiac biomarkers [8]. Our findings potentiate technological advancement towards POCT measurement of a very important cardiac biomarker, which can be employed in the outpatient setting and emergency departments especially when the hospitals do not have the capability for prompt laboratory sample processing. A further strength of our technology is its potential applicability to other well-established MI biomarkers such as $\mathrm{CK}-\mathrm{MB}$ and myoglobin, and recently emerging biomarkers such as ischemia- modified albumin (IMA) [24] or copeptin [25].

\section{Limitation of current available assays}

Devices currently implemented in many hospitals for the detection of MI biomarkers have limited accuracies in concentration detection compared to potentially more sensitive technology. The limitation may lead to worse overall outcome for patients presenting with ACS. Furthermore, currently implemented assays for cTnI measurement are not universally standardized, causing variation in the threshold at which a sample is deemed abnormal. This lack of standardization imposes particular disadvantages, as measurement on assays from different manufacturers and at different institutions is not reliably comparable. Currently implemented diagnostic assays require trained personnel for processing in dedicated laboratories. In contrast, our technology proposes an alternative approach to troponin detection, allowing for data acquisition at hospitals and in the field in a matter of only seconds after blood is drawn, without the need for extensivelytrained personnel.

\section{Comparison with other POCT approaches}

The difficulties of managing a patient with non-specific chest pain, despite employment of a Bayesian approach in order to choose subsequent diagnostic tools, fuels a general trend towards more sensitive and specific assays for the diagnosis of ACS. Similar quantitative and qualitative approaches for the detection of cTnI and other cardiac biomarkers for MI have been previously described in a recent comprehensive systematic review of POCT devices comprising 42 studies [8], with most described modalities principled on immunoassays. Despite these approaches, the review concludes that an optimal POCT for the timely diagnosis of ACS within 6 hours after the onset of symptoms does not yet exist [8]. In fact, recently published studies were deemed generally of suboptimal methodological quality and POCT devices reported an unacceptably high number of false negatives to be considered safe for the diagnosis of ACS [8]. This review, however, does highlight the necessity of novelty and technological evolution of this arena [8]. Compared to contemporary troponin assays [26-29], "high-sensitivity troponin" assays have demonstrated superior sensitivity, negative predictive value, and prognostic implications, but with a suboptimal positive predictive value $[30,31]$.

Qualitative based devices [32] are further inherently limited in that clinical interpretation of cardiac biomarkers are often complicated by an elevation related to non-cardiac causes of chest pain with classic examples pertaining to patients with renal impairment or pulmonary embolism. Moreover, chest pain is a common chief complaint. Its presenting symptoms can vary and correlate with a widely differing diagnoses in individuals with different risk factors. Such diversity in clinical presentations is more common in women and diabetic patients who have other physiological factors, which at times obscure the underlying etiology and delay the vital treatment. A quantitative approach is thus required since the extent of elevation of biomarkers often plays a key role in physicians' management decisions.

\section{Limitations}

Inherent limitations impede the practical implementation of our findings. While demonstration of reproducible results in the setting of both BSA as well as human serum is promising and physiologically similar to whole human blood, the identified CTnI signature needs to be corroborated within whole human blood and ultimately in vivo. The extremely low physiological concentrations of MI biomarkers and the confounding noise from other ubiquitous molecular structures within the blood create some technological challenges. In addition, despite structural similarities between human and mouse cTnI [12], our findings require corroboration using recombinant human $\mathrm{CTnI}$ protein prior to clinical implementation. Lastly, while scientifically feasible, a quantitative apparatus must be engineered, designed and verified for repeatability, accuracy, and specificity in the measurement of troponin concentrations in blood.

\section{Conclusion}

In conclusion, we herein present the unique Raman signature of mouse cardiac troponin I protein in Bovine Serum Albumin and Human Blood Serum. Future efforts to optimize this modality for the measurement of cardiac biomarkers could compensate for deficiencies in the currently utilized troponin measurement assay. Ultimately, this finding can assist in optimal diagnosis of patients suspected of presenting with ACS. 
Citation: Moghaddamjoo S, Tashakor AP, Fard ST, Tashakkor AY, Kwok E, et al. (2015) Characterization of Cardiac Troponin I Raman Signature in Bovine Serum Albumin and Human Blood Serum for the Potential Diagnosis of Myocardial Infarction. Nat Prod Chem Res 4: 200. doi:10.4172/2329-6836.1000200

Page 5 of 5

\section{References}

1. Jaffe AS, Babuin L, Apple FS (2006) Biomarkers in acute cardiac disease: the present and the future. J Am Coll Cardiol 48: 1-11.

2. Arenja N, Reichlin T, Drexler B, Oshima S, Denhaerynck K, et al. (2012) Sensitive cardiac troponin in the diagnosis and risk stratification of acute heart failure. J Intern Med 271: 598-607.

3. Celik S, Giannitsis E, Wollert KC, Schwöbel K, Lossnitzer D, et al. (2011) Cardiac troponin T concentrations above the 99th percentile value as measured by a new high-sensitivity assay predict long-term prognosis in patients with acute coronary syndromes undergoing routine early invasive strategy. Clin Res Cardiol 100: 1077-1085.

4. Anderson JL, Adams CD, Antman EM, Scott Wright R, Charles Bridges R, et al. (2013) 2012 ACCF/AHA focused update incorporated into the ACCF/AHA 2007 guidelines for the management of patients with unstable angina/non-STelevation myocardial infarction: a report of the American College of Cardiology Foundation/American Heart Association Task Force on Practice Guidelines. J Am Coll Cardiol 60: 645-681.

5. Alpert JS, Thygesen K, Antman E, Bassand JP (2000) Myocardial infarction redefined-a consensus document of the joint European Society of Cardiology/ American College of Cardiology committee for the redefinition of myocardial infarction. J Am Coll Cardiol 36: 959-969.

6. Santaló, M, Martin A, Velilla J, Povar J, Temboury F, et al. (2013) Using highsensitivity troponin $\mathrm{T}$ : the importance of the proper gold standard. Am J Med 126: 709-717.

7. Conroy PJ, O'Kennedy RJ, Hearty S (2012) Cardiac troponin I: a case study in rational antibody design for human diagnostics. Protein Eng Des Sel 25: 295-305.

8. Bruins Slot MH, van der Heijden GJ, Stelpstra SD, Hoes AW, Rutten FH (2013) Point-of-care tests in suspected acute myocardial infarction: a systematic review. Int J Cardiol 168: 5355-5362.

9. Perkins-Porras L, Whitehead DL, Strike PC, Steptoe A (2009) Pre-hospital delay in patients with acute coronary syndrome: factors associated with patient decision time and home-to-hospital delay. Eur J Cardiovasc Nurs 8: 26-33.

10. Ling Y, Pong T, Vassiliou CC, Huang PL, Cima MJ (2011) Implantable magnetic relaxation sensors measure cumulative exposure to cardiac biomarkers. Nat Biotechnol 29: 273-277.

11. Fard ST, Chrostowski L, Kwok E, Amann MC (2010) Chemometric approach for improving VCSEL-based glucose predictions. IEEE Trans Biomed Eng 57 $578-585$

12. Kho AT, Kang PB, Kohane IS, Kunkel LM (2006) Transcriptome-scale similarities between mouse and human skeletal muscles with normal and myopathic phenotypes. BMC Musculoskeletal Disorders 7: 1-9.

13. Lozano R, Naghavi M, Foreman K, Lim S, Shibuya K, et al. (2012) Global and regional mortality from 235 causes of death for 20 age groups in 1990 and 2010: a systematic analysis for the Global Burden of Disease Study 2010. Lancet 380: 2095-2128.

14. Talebi Fard, S, Grist, SM, Donzella V, Shon Schmidt A, Flueckiger J, et al. (2013) Label-free silicon photonic biosensors for use in clinical diagnostics. Proc SPIE 8629: 1-14.

15. Fard ST, Donzella V, Schmidt SA, Flueckiger J, Samantha Grist M, et al. (2014) Performance of ultra-thin SOI-based resonators for sensing applications. Opt Express 22: 14166-14179.

16. Talebi Fard S, Hofmann W, Talebi Fard P, G Bohm, et al. (2008) Optical absorption glucose measurements using $2.3 \mu \mathrm{m}$ vertical-cavity semiconductor lasers. IEEE Photonics Tech Lett 20: 930-932.

17. Talebi Fard S, Hofmann W, Talebi Fard P, Kwok E, Markus-Christian A, et al. (2009) Optical glucose monitoring using vertical cavity surface emitting lasers (VCSELs). SPIE Proceedings 7397: 1-14.
18. Burns DA, Ciurczak EW (2008) Handbook of Near-Infrared Analysis. (3rd Edn) Ser Practical Spectroscopy, Boca Raton, CRC Press, USA. p: 834.

19. Wise BM, Shaver JM, Gallagher NB, Windig W, Bro R, et al. (2006) Manual PLS Toolbox 4.0. Eigenvector Research Incorporated, Wenatchee, WA, USA

20. Li AY, Stevens CM, Liang B, Rayani K, Little S, et al. (2013) Familia hypertrophic cardiomyopathy related cardiac troponin C L29Q mutation alters length-dependent activation and functional effects of phosphomimetic troponin I. PLoS One. 8: 1-13.

21. Krüger M, Pfitzer G, Stehle R (2003) Expression and purification of human cardiac troponin subunits and their functional incorporation into isolated cardiac mouse myofibrils. J Chromatogr B Analyt Technol Biomed Life Sci 786: 287 296

22. Lee-Lewandrowski E, Laposata M, Eschenbach K, et al. (1994) Utilization and cost analysis of bedside capillary glucose testing in a large teaching hospital: implications for managing point of care testing. Am J Med 97: 222-230.

23. Tashakkor AY, Mancini GB (2013) The relationship between skin cholesterol testing and parameters of cardiovascular risk: a systematic review. Can $J$ Cardiol 29: 1477-1487.

24. Worster A, Devereaux PJ, Heels-Ansdell D, Gordon Guyatt $H$, John Opie, et al. (2005) Capability of ischemia-modified albumin to predict serious cardiac outcomes in the short term among patients with potential acute coronary syndrome. CMAJ 172: 1685-90.

25. Lipinski MJ, Escárcega RO, D'Ascenzo F, Magalhães MA, Baker NC, et al. (2014) A Systematic Review and Collaborative Meta-Analysis to Determine the Incremental Value of Copeptin for Rapid Rule-Out of Acute Myocardial Infarction. Am J Cardiol 113: 1581-1591.

26. Aldous S, Pemberton C, Richards AM, Troughton R, Than M (2012) Highsensitivity troponin $\mathrm{T}$ for early rule-out of myocardial infarction in recent onset chest pain. Emerg Med J 29: 805-810.

27. Weber M, Bazzino O, Navarro Estrada JL, de Miguel R, Salzberg S, et al (2011) Improved diagnostic and prognostic performance of a new highsensitive troponin T assay in patients with acute coronary syndrome. Am Heart J 162: 81-88.

28. Hochholzer W, Reichlin T, Twerenbold R, Stelzig C, Hochholzer K, et al. (2011) Incremental value of high-sensitivity cardiac troponin $\mathrm{T}$ for risk prediction in patients with suspected acute myocardial infarction. Clin Chem 57: 1318-1326.

29. Keller T, Zeller T, Ojeda F, Stergios Tzikas, Lars L, et al. (2011) Serial changes in highly sensitive troponin I assay and early diagnosis of myocardial infarction. JAMA 306: 2684-2693.

30. Mosleh W, Abdel-Qadir H, Farkouh M (2013) Biomarkers in the emergency workup of chest pain: uses, limitations and future. Cleve Clin J Med 80: 589598.

31. Reichlin T, Hochholzer W, Bassetti S, Stephan S, Claudia S, et al. (2009) Early diagnosis of myocardial infarction with sensitive cardiac troponin assays. N Engl J Med 361: 858-867.

32. Farsi D, Pishbin E, Abbasi S, Hafezimoghadam P, Fathi M, et al. (2013) Operating characteristics of a qualitative troponin assay for the diagnosis of acute coronary syndrome. Eur J Emerg Med 20: 120-122. 